\title{
EL CAMBIO CLIMÁTICO Y LA DEGRADACIÓN DEL SUELO: ESTUDIO DE CASO CUENCA ARROYO ESTACAS, PROVINCIA DE ENTRE RIOS, ARGENTINA
}

\author{
Nathalia Waked Sánchez*
}

\section{RESUMEN}

En los últimos veinte años, el departamento de La Paz ha tenido procesos vinculados al cambio en el uso de la tierra, aumentando los problemas de degradación del suelo. En este contexto, el objetivo del presente artículo de investigación es discutir si las acciones realizadas por el hombre, como el cambio en el uso de la tierra, la deforestación, entre otros, son causantes del problema de la degradación del suelo que presenta la cuenca, y si existe relación entre estos y el cambio climático. Entre los principales resultados, se destaca que las actividades agrarias realizadas, han sido uno de los causantes por las cuales se han incrementado los problemas de degradación, además, de aumentar las emisiones de dióxido de carbono, afectando con ello al suelo y el ambiente que los rodea.

Palabras clave: ambiente, cambio climático, degradación del suelo, producción agraria.

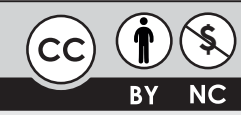

Recibido: 15 de noviembre de 2019 | Aprobado: 14 de febrero de 2020.

\section{CÓMO CITAR ESTE ARTÍCULO:}

Waked Sánchez, N. (2020). El cambio climático y la degradación del suelo: Estudio de caso Cuenca Arroyo Estacas, Provincia de Entre Ríos, Argentina. Collectivus, Revista de Ciencias Sociales, 7(2), 35-50. DOI: https://doi.org/10.15648/Collectivus.vol7num2.2020.2672

\footnotetext{
"Máster en Estudios Sociales y Agrarios. Facultad Latinoamericana de Ciencias Sociales (FLACSO), Argentina. Centro de Estudios de Sociología del Trabajo (CESOT), Facultad de Ciencias Económicas, Universidad de Buenos Aires. CP. 1120. Buenos Aires (Argentina). E-mail: nathalia.waked@fce.uba.ar
} 
36|El cambio climático y la degradación del suelo

\title{
CLIMATE CHANGE AND SOIL DEGRADATION: CASE STUDY OF CUENCA ARROYO ESTACAS, ENTRE RÍOS PROVINCE, ARGENTINA
}

\begin{abstract}
In the last 20 years, in the Department of La Paz There have been processes linked to the change in land use, which generated environmental impacts, increasing the risk of erosion. In this sense, the objective of this article is to discuss whether the actions carried out by man, such as the change in land use, deforestation, among others, are causing the problem of land degradation, and if there is a relationship between these activities and climate change. Among the main results, it is highlighted that the agricultural activities carried out, have been one of the causes for which the problems of land degradation have increased, in addition to increasing the emissions of carbon dioxide, nitrous oxide, methane, among others; radically changing the weather, thereby affecting the soil.
\end{abstract}

Keywords: ambient, agricultural production, climate change, Soil degradation.

\section{Introducción}

En el departamento de La Paz (Provincia de Entre Ríos), el problema de la degradación del suelo es cada vez más significativo, el cual se vincula especialmente con el cambio en el uso de la tierra que se ha producido en los últimos veinte años. En este sentido, el propósito del presente trabajo es discutir si existe relación entre el cambio climático y el problema de la degradación del suelo y si las acciones realizadas por el hombre, como el cambio en el uso de la tierra, la deforestación, entre otras, incidieron sobre esta situación.

En la primera parte del artículo, se expone brevemente el contexto de la región, explicando cómo ha sido el proceso de degradación en la zona y los diferentes periodos agrícolas que allí se han presentado, con el objetivo de entender la relación que hay entre el avance de la agricultura y el aumento de la degradación del suelo. En la segunda parte, a partir del enfoque de la sociología ambiental, se estudia la relación entre el cambio climático y el problema de la degradación, a partir del análisis de la acción humana como sujeto que ha intervenido la historia y perturbado a la naturaleza, y desde el papel histórico que el medio físico ha tenido en la manifestación de los problemas ambientales. Este apartado está dividido en dos: el primero estudia el impacto del cambio climático en la agricultura, y el segundo hace un análisis sobre la relación entre cambio climático, agricultura y degradación del suelo.

Este artículo, se deriva de la tesis de maestría Estrategias para enfrentar el problema de la degradación de la tierra por parte de productores agrícolas, realizada durante los años 2017 y 2018. La zona de estudio, está ubicada en la cuenca arroyo Estacas, departamento de La Paz, provincia de Entre Ríos, Argentina; escogida por ser una zona en donde los problemas de degradación del suelo han sido considerables, convirtiéndose en un gran inconveniente tanto para la sustentabilidad ambiental como para la producción agraria. 


\section{Metodología}

Como estrategia metodológica, el artículo se sitúa dentro del estudio de caso con un abordaje metodológico cualitativo, permitiendo acercarse a los fenómenos sociales privilegiando su profundidad, lo que resulta adecuado para un problema de investigación que posee varias dimensiones a analizar. Igualmente, se combinó con procedimientos cuantitativos, así como datos y análisis de fuentes secundarias.

Para el presente artículo, se hizo uso de los resultados generados en el trabajo de campo del proyecto de investigación: Estrategias para enfrentar el problema de la degradación de la tierra por parte de productores agrícolas, donde las técnicas de investigación empleadas, fueron entrevistas a productores agrícolas y ganaderos ubicados en la cuenca, técnicos de la Estación Experimental de INTA (sedes Paraná, La Paz y Crespo), y técnicos particulares y personas vinculadas con los gobiernos provinciales y municipales expertos en el tema ${ }^{1}$. Además, se tuvieron en cuenta dos relevamientos ${ }^{2}$ de información cuantitativa que el INTA Paraná realizó a productores familiares de la cuenca: una enfocada en la adopción de prácticas de Manejo Sostenible de Tierras (MST), y la segunda se realizó con el objetivo de relevar datos para el análisis de indicadores socioeconómicos ${ }^{3}$; además de información secundaria. Para el análisis de datos, a partir de la información recopilada, se establecieron cinco ejes centrales: ambiente, degradación del suelo, agricultura familiar, estrategias y saberes, para así analizar la información y organizarla por medio de estos. Por último, se comenzó con el proceso de escritura.

\section{Contexto de la región}

Uno de los problemas ambientales más significativos en los últimos años, en la región del Noroeste de la provincia de Entre Ríos, es el de la degradación del suelo, como el producto de complejas interacciones de factores naturales y humanos, en donde "el uso inadecuado de los recursos naturales por parte del hombre ha conducido a la pérdida irreversible de la productividad de las tierras y los servicios ecosistémicos, con un consecuente impacto negativo sobre las poblaciones que de ellos dependen" (Aranda, 2004). La escasez del agua en los periodos de sequía y las altas precipitaciones en los periodos de lluvia, han agravado los procesos de degradación, que, si no tienen un manejo adecuado, pueden dar lugar a procesos de desertificación. Así lo explican Arnulfo Encina Rojas y José Ibarra (2003), al exponer sobre las consecuencias que puede llegar a tener procesos de erosión, como es la desertificación:

En los últimos decenios, la ganadería y el pastoreo excesivo han ocasionado daños incalculables en numerosas regiones de África, Asia y América Central y del Sur. En las zonas más secas, el resultado final de la erosión es la desertificación. Si la erosión es para la tierra una enfermedad, la desertificación significa su muerte. Hoy, la desertificación amenaza a unos 3.200 millones de personas en el mundo (Encina \& Ibarra, 2003, p.7).

\footnotetext{
${ }^{1}$ En cuanto a la elección de los productores a entrevistar, se basó en el criterio de los técnicos extensionistas que estaban a cargo del proyecto Soporte a la toma de decisiones para la ampliación e integración del manejo sustentable de tierras.

${ }^{2}$ El relevamiento de información utilizado, es una herramienta creada para registrar información específica sobre una situación o problema a observar.

${ }^{3}$ El primer relevamiento cuantitativo, se aplicó a productores que fueron convocados a un taller del INTA, en conjunto con la Cooperativa de La Paz y la Subsecretaría de AF, relevándose un total de 45 cuestionarios. Para el segundo, se procesó información proveniente del Relevamiento a Productores realizado por el Observatorio Nacional de Degradación de Tierras y Desertificación (http://www.desertificacion.gob.ar/wp-content/uploads/2018/03/ONDTyD_inf-preliminar12-171.pdf).
} 
38|El cambio climático y la degradación del suelo

En los últimos decenios, la ganadería y el pastoreo excesivo han ocasionado daños incalculables en numerosas regiones de África, Asia y América Central y del Sur. En las zonas más secas, el resultado final de la erosión es la desertificación. Si la erosión es para la tierra una enfermedad, la desertificación significa su muerte. Hoy, la desertificación amenaza a unos 3.200 millones de personas en el mundo (Encina \& Ibarra, 2003, p.7).

En los últimos veinte años, en el departamento de La Paz se han identificado procesos vinculados al cambio en el uso de la tierra, aumentando los problemas de erosión, afectando la producción agrícola y ganadera. Para la región del Noroeste de la provincia, la degradación del suelo se produce como consecuencia de la erosión, afectando su fertilidad. Por ello, en seguida, se analizará la relación entre las características del paisaje con los problemas de degradación propios de esta zona.

El paisaje, se distingue por tener gran cantidad de relieves con pendientes importantes y un régimen pluviométrico elevado, haciendo que el potencial erosivo por causa de las pendientes y de la intensidad de la lluvia sea alto. Al encontrase la región, entre dos grandes ríos el Uruguay y el Paraná toda su superficie está surcada por arroyos, ríos, nacientes, favoreciendo el problema de erosión hídrica, que es el tipo de erosión que afecta en mayor medida a esta zona de la provincia.

En cuanto a los suelos, estos se encuentran bajo relieves con pendientes pronunciadas y su característica arcillosa favorece el proceso erosivo, entonces, ante una fuerte precipitación se generan profundas cárcavas, especialmente cuando el suelo está labrado. Específicamente, en la cuenca arroyo Estacas se encuentran suelos vertisoles, con una alta proporción de arcilla expandible, de baja capacidad de drenaje interno y superficial, y deficientes en fósforo. Los suelos están formados por un material fino y loéssico, muy susceptible a la erosión hídrica.

A continuación, se muestra el resultado del relevamiento de suelos de la Provincia de Entre Ríos, explicando las características de los suelos presentes en esta región, así como su predisposición a procesos de degradación:

A partir de los inicios del relevamiento de suelos en la provincia de Entre Ríos (Convenio INTA-Gobierno de Entre Ríos, 1969-2011) fueron reconocidos cinco órdenes: Vertisoles, Molisoles, Alfisoles, Entisoles e Inceptisoles (Plan Mapa de Suelos, 1974). Aproximadamente, el $70 \%$ de la superficie de tierra firme presenta suelos con características vérticas (vertisoles y suelos molisoles asociados). La baja capacidad de infiltración de estos suelos debido a sus características intrínsecas y la topografía ondulada, con pendientes pronunciadas ( $1 \mathrm{a} 4 \%$ ), predisponen a gran parte de la superficie provincial a procesos de degradación de suelos, especialmente por erosión hídrica (Casas y Albarracin, 2015, citando a Scotta et al., 1989, p.112).

El proceso de erosión hídrica, que se ha presentado en la cuenca es el resultado de suelos que infiltran poco el agua, debido al alto contenido de arcilla, precipitaciones muy intensas y pendientes del suelo pronunciadas, más la extracción cada vez más intensa de la cobertura vegetal hecha por el hombre. En los períodos con mucha lluvia, el agua va generando surcos que aumentan su tamaño al incrementar el agua arrastrando la mayoría de los nutrientes. Luego, los surcos se van convirtiendo en pequeñas cárcavas que con el aumento de la lluvia crecen hasta romper completamente la superficie; situación que es analizada en una de las entrevistas realizadas a uno de los productores que vive en la cuenca:

"El problema en esta zona, son las pendientes que lleva la tierra, y nosotros tenemos que prevenir que no se degraden, digamos un poquito también por el tema de los desmontes. Se desmontaron lugares donde corría el agua, hacia los afluentes que hoy no se deberían haber desmontado, entonces las tierras buenas se están yendo hacia los afluentes y con ello se van los nutrientes, por eso básicamente, tenemos que volver a pensar en mucha cobertura de materia seca, materia orgánica arriba de los campos y no aflojar en ese sentido, también curvas de nivel, con el objetivo de que no tome velocidad el agua con las lluvias grandes, conservando la estructura y nutrientes de la tierra". (Productor A, Cuenca Arroyo Estacas, Entre Ríos, Argentina, 20 de octubre de 2017). 
De tal forma, que, con el avance de la agricultura y la ganadería, las aguas han estado más expuestas a contaminarse, debido a que cuando llueve en grandes proporciones hay arrastre de partículas y escurrimiento, produciendo cambios en el ambiente acuático, alterándolo. Es decir, la degradación del suelo no sólo produce una disminución en la rentabilidad de la producción agrícola, sino que también puede afectar el medio que la rodea.

Los pastizales naturales, representan el principal recurso forrajero de los sistemas ganaderos de cría, y constituyen el estrato herbáceo del bosque semi xerofítico (bosque o monte nativo) de la provincia fitogeográfica del Espinal, compuesto por especies de la flora pampeana, en su mayoría gramíneas estivales, de buen valor forrajero (Lezana, Litwin, Mancuso y Pueyo, 2012, citando a Cabrera, 1976). Sin embargo, al ser removida la mayoría de la vegetación nativa presente en el monte, ha surgido el espinal caracterizado por presentar pastizal por debajo (Técnico A, Departamento de La Paz, Entre Ríos, Argentina, 19 de octubre de 2017). Por lo tanto, la remoción de la vegetación nativa en la mayor parte de la región, ha sido una de las causantes que ha incrementado dicho problema.

Concretamente, el proceso de degradación que ha experimentado la cuenca, se acrecentó en consecuencia de la remoción de la capa arbórea por causa de la ganadería, la deforestación, los periodos agrícolas de intensa producción (factores humanos) y las características erosivas de sus suelos (factores naturales). Antes de los años noventa, el suelo se conservaba relativamente sano, pero con la intensificación de la agricultura en los últimos veinte años, produjo cambios importantes como la eliminación de la mayoría de los arbustos y pastizales, la deforestación, entre otros factores ya indicados, haciendo que el proceso sea cada vez más preocupante. Por ello, para una mayor comprensión de esta situación, se explicarán los diferentes periodos agrícolas registrados en la cuenca.

\subsection{Cambios en el uso de la tierra en la cuenca arroyo Estacas}

La cuenca arroyo Estacas, fue poblada por criollos y migrantes descendientes especialmente de colonias francesas e italianas, que arribaron al país desde finales de siglo XIX y comienzos del siglo XX. Mientras que la población criolla, nacida en la misma región o en regiones vecinas como Santa Fe, Buenos Aires y Corrientes migró a esta zona principalmente para obtener tierra.

En cuanto al uso que hacían de la tierra, en aquel momento uno de los productores entrevistados recordaba: "Por aquella época se producía más que todo ganadería y maíz, pero en pequeña escala, especialmente para darle de comer a las aves, a las gallinas y harina para consumo de la casa" (Productor A, Cuenca Arroyo Estacas, Entre Ríos, Argentina, 20 de octubre de 2017), observándose en el relato la preeminencia de la actividad ganadera.

Marcelo Wilson, técnico del INTA Paraná, relataba que desde la época de Hernandarias siglo XVI, la entrada de gran cantidad de vacas a la zona, se convirtió en uno de los antecedentes que influyó en la transformación del monte natural, modificando lo que habían sido alguna vez montes prístinos. Para finales del siglo XIX, un hecho que marcó el ecosistema de esta zona fue la tala y desmonte del bosque nativo, suceso que se repitió entre las décadas de los años cincuenta y sesenta, especialmente para la construcción del ferrocarril.

Se conoce que, en la región entrerriana las primeras prácticas agrícolas se basaban en el arado como principal herramienta de labranza. Para principios del siglo XX, periodo en la que llegaron la mayoría de los migrantes de origen europeo, se decía que los suelos ya presentaban problemas erosivos. Para esa época, la forma de trabajo que se usaba en 
40| El cambio climático y la degradación del suelo

la agricultura, era el de arado con bueyes, método que afectaba el suelo, pero como el manejo no era intensivo como el actual, su grado de afectación era bajo.

A mediados de siglo XX, décadas de los años cuarenta y cincuenta, se empieza a implementar el uso de tractores y a tecnificarse más los métodos de siembra y cosecha, incrementando los niveles de cultivo; ya en este periodo se empiezan a registrar niveles de erosión importantes. Existía una combinación entre la producción de maíz, trigo y ganadería y luego, a partir de los años sesenta, aumenta la creación de tambos para la producción de leche.

El proceso de degradación del suelo, en esta época ocurría a un ritmo mucho menor de lo que sucede en la actualidad, especialmente porque la maquinaria no tenía la capacidad ni la tecnología que existe ahora. En relación con este contexto, uno de los productores entrevistados, relata brevemente los procesos de transformación en la producción agropecuaria presentados en la cuenca:

"Yo nací acá, en el año setenta y cuatro. En los años setenta había mucha agricultura, muchísima, porque en la década del ' 60 y '70, se hicieron bastantes lotes agrícolas a través del desmonte. Para fines de los años '70, la agricultura era muy intensiva, pero en la década del '80, el proceso de la agricultura empezó a decaer... fue una época muy mala". (Productor D. Cuenca Arroyo Estacas, Entre Ríos, Argentina, 22 de octubre de 2017).

Con el paso de los años, como se comenta, especialmente en los años setenta, el uso del suelo se intensificó, y al aumentar el uso de este, se empezó a manifestar con mayor intensidad el problema de degradación ${ }^{4}$. Para los años ochenta, el porcentaje que había de agricultura en comparación con la ganadería era muy inferior, sin embargo, la continuidad de la actividad ganadera fue igualmente afectando los suelos. Así es descrito por uno de los productores:

"Mi mamá nació acá y mi papá vino de Santa Fe, con una cultura más abierta, más agrícola. Allá en el año 82 , más o menos tenían $50 \%$ de agricultura y $50 \%$ de ganadería, acá en Entre Ríos éramos $10 \%$ de agricultura y $90 \%$ de ganadería, los suelos allá eran más fértiles y tenían mejores tierras" (Productor A. Cuenca Arroyo Estacas, Entre Ríos, Argentina, 20 de octubre de 2017).

En resumen, la producción ganadera ${ }^{5}$, se mantuvo alternando momentos de mayor producción con otros de menor producción, por consiguiente, siempre conservó su importancia. Luego, en la década de los noventa empieza a tomar cada vez más fuerza el cultivo de soja. Actualmente, ha disminuido en gran medida la producción de soja, reconvirtiéndose la región a la ganadería, debido especialmente, a la sequía que hubo en la temporada de verano 2017-2018, y porque los precios de la producción de carne mejoraron en los últimos años, aumentando su exportación.

En la zona donde queda ubicada la cuenca arroyo Estacas, el fenómeno del cambio en el uso de la tierra registrado en los últimos años fue notorio, gracias a la conversión de ecosistemas prístinos a cultivados y la simplificación de los esquemas de rotaciones en tierras agrícolas con tendencias al monocultivo de soja, en donde se recurrió a la práctica de la deforestación, en la mayoría de los casos sin planificación previo del uso y manejo del suelo (Dupleich y Vicente, 2012).

\footnotetext{
${ }^{4}$ Especialmente, porque requiere poca inversión en comparación con otros sistemas ganaderos.

${ }^{5} \mathrm{La}$ cuenca posee tierras aptas para planteos productivos en rotación ganadero-agrícolas. No obstante, la mayor superficie presenta aptitud ganadera. Por ello, el sistema de uso predominante ha sido el ganadero de cría en base al campo natural bajo monte nativo. Los bosques nativos constituyen la base productiva de los establecimientos agropecuarios ganaderos, mayoritariamente de cría bovina (Lezana, Litwin, Mancuso y Pueyo, 2012).
} 
Es importante tener en cuenta, que durante los años setenta surge la revolución verde, aumentando los niveles de producción e incentivando la implementación de agroquímicos en la agricultura; suceso que influyó a nivel mundial en todo el sector agropecuario, modificando las formas de producción. Desde aquel momento, la producción agrícola especialmente el monocultivo de soja, aumentó considerablemente en la mayoría de las zonas de producción agraria en Argentina, así como en la zona de estudio.

En conclusión, los factores que más incidieron en el aumento del problema de degradación del suelo, durante el siglo XX fue la intensificación de los procesos productivos, donde con el paso de los años la tecnificación y la maquinaria afectó cada vez más el suelo. La falta de un método que ayudará a recuperarlo, luego del uso intensivo del cultivo de soja, además del desmonte del monte natural, la eliminación del monte prístino y la continuación en la implementación de la ganadería, más una predisposición natural de los suelos a la erosión, fueron suficientes para que dicho problema se acrecentara.

Los cambios en el uso de la tierra, registrados en la cuenca arroyo Estacas, son una manifestación de cómo el avance de la agricultura y ganadería, contribuyó en la intensificación de los problemas de degradación del suelo presentes en la zona. Pero, para entender por qué se fue desarrollando este proceso, en el siguiente apartado se va a presentar brevemente la perspectiva teórica de la sociología ambiental, para después analizar la relación entre el cambio climático, la agricultura y el proceso de degradación.

\section{El cambio climático: una mirada desde la sociología ambiental}

La sociología ambiental, surge a mediados de los años setenta en Estados Unidos, entre sus precursores se encuentra Dunlap y Canton. Su objetivo, era estudiar las complejas y variadas interacciones que se daban entre la sociedad y el ambiente, para ello utilizaron el concepto "complejo ecológico". Desde su inicio hasta la actualidad, la sociología ambiental ha tenido gran cantidad de variaciones, por lo tanto, para esta ocasión se va a hacer énfasis de una sociología ambiental que tiene como propósito deconstruir los paradigmas científicos derivados de la racionalidad moderna (Leff, 2010).

Para la sociología ambiental, la crisis ambiental no se reconoce como un hecho natural, resultado de la evolución natural del mundo, sino como una acción humana que ha intervenido a la historia y perturbado a la naturaleza (Leff, 2010). El autor plantea, que el origen de la crisis es antropogénico, es más, que la instauración y la institucionalización de una racionalidad social moderna, ha conducido a la insustentabilidad ambiental por acción exclusiva del hombre.

En consecuencia, la destrucción eventual de los ecosistemas naturales, ha influido en el surgimiento de fenómenos como la degradación del suelo, actuando desfavorablemente sobre las posibilidades agropecuarias ulteriores derivados de la simplificación de los ecosistemas puestos en explotación. En este sentido Claudia E. Natenzon y Gustavo Tito (2001), comentan:

La mayor o menor intensidad con la que se use la tierra, la mayor o menor selectividad de las actividades económicas emprendidas y otros factores que pueden afectar a los recursos naturales y el ambiente; van a estar determinados por los factores que determinan esos niveles de rentabilidad diferentes. Muchos fenómenos de erosión de suelos, prácticas de manejo intensivas, contaminación de aguas, etc.; encuentran su explicación en estos procesos. (Natenzon \& Tito, 2001). 
Como se explica en la cita, el deterioro de los recursos se demuestra por el nivel de intensidad con la que se ha usado la tierra, y por la selectividad de las actividades económicas emprendidas. Es decir, el deterioro de los recursos depende si el productor realiza una producción intensiva, o si su actividad ha tenido un manejo adecuado ambientalmente, en ese caso el grado de afectación sobre los recursos naturales es diferente. Ahora, si el productor presenta un uso intensivo en su explotación, los niveles de rentabilidad son mayores, así como el deterioro sobre los recursos naturales, mientras que una actividad donde el manejo de éstos sea más moderado, el deterioro será menor. Según esto, a continuación, se explicará porque el cambio climático podría estar relacionado con la racionalidad social moderna.

De acuerdo con el problema del cambio climático, el autor Richard B. Norgaard ${ }^{6}$ comenta que este debe ser analizado desde una visión social y ambientalmente histórica, es decir, desde el análisis de indicios de procesos naturales en épocas pasadas ${ }^{7}$. Para ello, el estudio de la sociología ambiental, debe considerar el papel histórico del medio físico en la manifestación de los problemas ambientales en todo tipo de sociedades. Para lograr obtener esta información Richard B. Norgaard expone la metodología de la "coevolución":

El enfoque coevolucionista de la sociología del ambiente, permite explorar en qué se diferencian las sociedades modernas y las no modernas, proporciona una explicación de los modos en que la gente influye en su entorno y el entorno influye en las sociedades con el paso del tiempo, y se puede utilizar también para documentar cultural y ambientalmente historias locales y concretas. (Ayestarán, 2004, p.114).

Richard B. Norgaard, argumenta que el cambio climático se produce por la variación de variables climáticas, de acuerdo con los siguientes factores: calentamiento de la atmosfera, disminución de la extensión de nieve y ascendencia del nivel del mar, más otros cambios observados, como aumento de las precipitaciones, repetición más frecuente del fenómeno del Niño, sequías más intensas, y por último aumento en la concentración de gases de efecto invernadero. ${ }^{8}$

Ahora bien, para analizar que los cambios efectuados en los últimos años en el clima, se diferencian de variaciones naturales de la tierra en periodos largos, es decir, que el cambio en el clima no se ha producido por una causa natural, que se haya presentado en periodos anteriores en la historia del planeta tierra, la sociología ambiental hace uso de la paleoclimatología, la cual utiliza diferentes tipos de indicadores como: documentos históricos, corales, polen fósil, anillos de árboles, testigos de hielo, sedimentos lacustres y

${ }^{6}$ El análisis de Richard B. Norgaard se relaciona con la sociología ambiental, pero desde una perspectiva menos radical respecto al análisis del impacto de la actividad humana en el ambiente. ${ }^{7}$ El calentamiento global, se produce por efecto del incremento de la concentración de GEI en la atmósfera, los que retienen el calor emitido desde la superficie del planeta. Los principales gases involucrados son el dióxido de carbono $(\mathrm{CO} 2)$, el metano $(\mathrm{CH} 4)$ y el óxido nitroso (N2O). (Caviglia, Wingeyer, Novelli, 2016, p.28).

${ }^{8}$ De acuerdo a la 3ra Comunicación Nacional de Cambio Climático (CIMA, 2015), las principales variables afectadas por cambio climático con impacto potencial sobre los suelos son: i) el incremento de la temperatura media explicado principalmente por mayores temperaturas nocturnas; ii) cambios en el régimen de precipitaciones en cuanto a distribución y cantidad; iii) el incremento en la ocurrencia de eventos climáticos extremos como sequías prolongadas, olas de calor o frío e inundaciones y iv) el aumento del $\mathrm{CO} 2$ atmosférico (Caviglia, Wingeyer, Novelli 2016, pp.29-30). 
oceánicos, entre otros. En este marco, Ignacio Ayestarán Úriz explica como por medio del registro paleoclimático se puede observar las fluctuaciones de la temperatura a nivel global, con el objetivo de comprender las causas del calentamiento a través del tiempo:

Los datos paleoclimáticos, proveen una confirmación independiente de este calentamiento reciente, y también ubican el calentamiento del siglo XX y XIX (de 1860 al presente), en el contexto de los últimos siglos y del último milenio. El registro paleoclimático, no sólo nos permite ver las fluctuaciones de la temperatura global a lo largo de los últimos siglos, sino que también permite a los científicos examinar el clima del pasado aún más lejos en el tiempo. Esta perspectiva, es una capacidad importante en nuestra búsqueda para comprender las posibles causas del calentamiento global en el siglo XX. Podemos mirar los supuestos periodos cálidos en el pasado distante (por ej. 1.000, 6.000, 125.000, e incluso 165.000.00 años en el pasado) para tratar de encontrar indicios de procesos naturales. Hasta ahora los paleoclimatólogos no han podido encontrar ninguna explicación climática natural para el calentamiento actual. (Ayestarán 2004,p.121).

Una las conclusiones a las que este autor ha llegado es que alrededor de las tres cuartas partes de las emisiones de $\mathrm{CO} 2$ antropogénicas, que se han producido en los últimos veinte años se debe a la quema de combustibles fósiles, el resto se debe fundamentalmente a los cambios en el uso de la tierra, especialmente, a la deforestación (Ayestarán, 2004).

Uno de los organismos que ha realizado evaluaciones periódicas, de los cambios climáticos a nivel global, es el Grupo Intergubernamental de Expertos sobre Cambio Climático, constituido en el año 1988, por la Organización Meteorológica Mundial OMM y el Programa de Naciones Unidas para el Medio Ambiente PNUMA. El objetivo principal del IPCC, (por sus siglas en inglés Intergovernmental Panel on Climate Change) es la de evaluar la información científica, técnica y socioeconómica relevante para la comprensión de las causas y efectos del cambio climático, así como de las alternativas para la lucha frente a estos cambios (op. cit). El IPCC no investiga, sólo recopila y evalúa la información publicada, emitiendo Informes de evaluación, e informes especiales o técnicos. Desde su creación son reconocidos tres informes.

El Primer Informe de Evaluación fue realizado en 1990, el cual sirvió como base para la creación de la Convención Marco de Naciones Unidas sobre Cambio Climático. El Segundo Informe, se realizó en 1995 y proporcionó información clave para las negociaciones que condujeron a la adopción del Protocolo de Kyoto (1997), y el Tercer Informe de Evaluación, se hizo en el año 2001. Los tres Informes de Evaluación, concluyen que en el último siglo los factores naturales han influido escasamente en el balance neto de energía en el sistema planeta-atmósfera. Estos informes del IPCC, presentan evidencias nuevas y más poderosas que el calentamiento observado en los últimos cincuenta años es debido a las actividades humanas (op. cit). Informes que coinciden con lo obtenido en los datos paleoclimáticos. Conforme con lo expuesto, a continuación, se discutirá la relación entre la actividad agropecuaria y el cambio climático.

\subsection{Impacto del cambio climático en la actividad agropecuaria}

En cuanto a la relación entre el cambio climático y la actividad agropecuaria, en efecto se observa que la actividad agropecuaria, es uno de los sectores que genera emisiones de Gases de Efecto Invernadero GEI. En Argentina, para el año 2000 la ganadería aportó más del $30 \%$ de las emisiones totales producidas por actividades humanas en el 
país. La producción de bovinos para carne y leche es responsable de aproximadamente el 95\% de las emisiones del sector y el 5\% restante a todas las demás especies de producción (ovinos, caprinos, porcinos, equinos, aves, búfalos, asnales, mulares y camélidos sudamericanos), (S. de Obschatko, 2015). Otra de las acciones humanas, vinculadas con la actividad agropecuaria es la deforestación, a la cual muchos organismos hoy en día le están prestando creciente atención.

A nivel mundial, el informe del Grupo de Trabajo III del Quinto Informe del IPCC, que se dedica a analizar el grupo de agricultura, bosques y otros usos de tierra, denominado AFOLU por sus siglas en inglés (Agriculture, Forestry and Other Land Use), afirmaba que: el sector AFOLU es responsable por algo menos de un cuarto (alrededor de 10 a 12 GtCO2eq/año) de las emisiones de GEI, principalmente por la deforestación y las emisiones de la agricultura, ganadería y el manejo de nutrientes; la degradación forestal por la acción humana y la quema de biomasa también representan contribuciones importantes (op. cit, p.17). Es fundamental mencionar que, aunque el sector agrícola y ganadero influye en los GEI, en comparación con otros sectores, como por ejemplo el sector energético, su aporte es menos significativo. Sin embargo, en cuanto a las acciones que los organismos internacionales desarrollan para la reducción de los GEI, certifican que cada vez la agricultura toma mayor relevancia.

La actividad agropecuaria, ingresó formalmente a las discusiones en la Conferencia de las Partes COP el 17 del año 2011, en Durban, (Sudáfrica) en el marco de la Convención de las Naciones Unidas sobre Cambio Climático CMNUCC, durante el desarrollo de esta, se solicitó la creación del grupo de trabajo en agricultura bajo el Órgano Subsidiario de Asesoramiento Científico y Tecnológico (SBSTA, por sus siglas en inglés). Suceso que quedó registrado en la reunión No. 38 del Órgano Subsidiario de Asesoramiento Científico y Tecnológico (SBSTA), como se puede observar:

En junio de 2013, durante la reunión número treinta y ocho del Órgano Subsidiario de Asesoramiento Científico y Tecnológico (SBSTA), en sus siglas en inglés Subsidiary Body for Scientific and Technological Advice, se invitó a los países y organizaciones observadoras a presentar a la secretaría de la CMUNCC su punto de vista sobre "el estado actual de los conocimientos científicos, sobre la manera de incrementar la adaptación de la agricultura a los impactos del cambio climático a la vez que se promueva el desarrollo rural, el desarrollo sostenible, la productividad de los sistemas agrícolas y la seguridad alimentaria en todos los países, en particular en los países en desarrollo, tomando en cuenta la diversidad de los sistemas agrícolas, las diferencias en escalas y los posibles cobeneficios de la adaptación" (S. de Obschatko 2015, citando a UNFCCC, 2013, p.21).

Para los organismos internacionales, el tema de la agricultura presenta algunas contradicciones, ya que, por un lado, se encuentra la problemática de las emisiones de GEI que esta produce, pero al mismo tiempo, la agricultura cumple un rol clave en materia de seguridad alimentaria, creación de empleo y sustento económico para las poblaciones rurales con menos recursos, además de contribuir a la producción de energía (biocombustibles). Por lo tanto, dichos organismos han desarrollado estrategias con el objetivo de incorporar en sus proyectos estos dos escenarios, tratando de no afectar el uno al otro:

De acuerdo con la FAO (2013), los países estarían considerando crecientemente implementar acciones de mitigación en el sector agropecuario por dos razones fundamentales. Por un lado, por la contribución sectorial a las emisiones de GEI corrientes y esperadas (futuras) a nivel nacional. Por el otro, por las sinergias existentes entre la reducción de emisiones agrícolas y una serie de objetivos de desarrollo, fundamentalmente, seguridad alimentaria, adaptación a los cambios climáticos esperados, reducción de la deforestación, aumento de 
eficiencia y competitividad, promoción del acceso rural a fuentes energéticas, reducción de la contaminación del agua, restauración de tierras degradadas y aumento de la eficiencia en el uso de los recursos. (op. cit, p.20).

Gabriel Rodríguez, experto en Cambio Climático del INTA, comenta sobre esta situación:

"Cuando hablamos de cambio climático el componente de la actividad humana es un factor clave y asume la forma de tendencia creciente, principalmente en la temperatura global del planeta" [...] "Debemos implementar acciones para la disminución de las emisiones de gases de efecto invernadero y avanzar en el desarrollo de estrategias de adaptación de los cultivos que nos permitan mitigar los efectos". (Sommantico, 2018).

En conclusión, lo más importante para analizar sobre el cambio climático, es que las acciones realizadas por el hombre, como el cambio en el uso de la tierra, la deforestación, entre otros, son causantes de este. Situación que no sólo es resultado de una visión socialmente histórica, sino también por procesos ambientales que se han registrado en un mismo periodo, y que en épocas anteriores no habían sucedido de igual forma e intensidad; el cual puede ser analizado por medio de los datos paleoclimáticos, como de los Informes de Evaluación del IPCC, demostrando en efecto que estos cambios en el clima, se han acelerado en los últimos años a un ritmo nunca visto en la historia de los procesos naturales del planeta tierra. En últimas, tal como se describe en el artículo Mujer y medio ambiente. Una aproximación desde la acción socioeducativa "el ser humano se encuentra en constante interrelación con su entorno. Cuando este le produce algún perjuicio es que se habla de problemas ambientales" (Esteban, Amador, Mateos y Olmedo, 2019). No obstante, en el estudio del cambio climático, es importante tener en cuenta que alrededor de esta problemática existen posiciones dentro del mundo científico ajenas a este enfoque teórico, las cuales declaran que, en lugares específicos afectados por procesos como la degradación del suelo, este no estaría relacionado con el cambio climático.

\section{2.¿Existe relación entre cambio climático, agricultura y degradación del suelo?}

Una de las situaciones que es importante examinar, es si hay relación entre cambio climático, la agricultura y el problema de degradación del suelo. En la sociología ambiental, la interacción entre el ambiente y la sociedad, como se explicó anteriormente, plantea la perspectiva de la "coevolución". En el documento "De la historia y la sociología ambientales a la ecología política: factores tecnocientificos, sociohistóricos y ecosistémicos en la investigación sobre el cambio climático" de Ignacio Ayestarán Úriz, se llega a la conclusión de que en el último siglo los factores naturales poco han influido en el balance neto de energía en el sistema planeta-atmosfera, evidenciando así que el calentamiento observado en los últimos cincuenta años es debido mayormente por actividades humanas:

El estudio del clima en épocas anteriores también nos ayuda a comprender de qué forma el ser humano está influyendo en el cambio climático del planeta. El registro climático de los últimos mil años muestra claramente que las temperaturas globales aumentaron significativamente en el siglo XX, y que este calentamiento no tiene prácticamente precedentes en los últimos mil doscientos años. (Ayestarán, 2004, p.126).

Resultado que se obtiene a partir del registro paleoclimático, utilizando diferentes tipos de datos como: documentos históricos, corales, polen fósil, anillos de árboles, testigos de hielo, sedimentos lacustres y oceánicos, entre otros. Este registro puede llegar a 
46|El cambio climático y la degradación del suelo

analizar los periodos cálidos de hasta 165.000.000 años en el pasado, tratando de encontrar indicios de procesos naturales que pudiesen estar causando el calentamiento global que ahora experimentamos (op. cit), indicando que hasta el momento no han encontrado indicios de procesos naturales que expliquen el calentamiento actual.

Al hablar con los productores del departamento de La Paz, la gran mayoría comentaban acerca de los cambios que han percibido en el clima, del incremento de las lluvias y de la temperatura, como lo relata una de las personas entrevistadas:

"Para mí que por ahí veinticinco años atrás se han visto cambios, que yo recuerde, cuando yo era chico los calores no eran iguales. Antes la lluvia era más normal, ahora tanto el calor como las lluvias son más intensos. Los calores... se nota que no eran los calores de antes, porque ahora son insoportables". (Productor A, Cuenca Arroyo Estacas, Entre Ríos, Argentina, 20 de octubre de 2017).

Otro productor mencionaba: "la temperatura ha cambiado enormemente, los veranos son mucho más bravos que antes, porque antes 30 grados eran soportables, ahora 40 grados y húmedo, no se soportan, ese calor húmedo en el verano es fatal" (Productor B, Cuenca Arroyo Estacas, Entre Ríos, Argentina, 21 de octubre de 2017). Los relatos de los productores, en efecto demuestran la preocupación por el aumento de las lluvias y del clima, especialmente porque afecta directamente su actividad económica.

Específicamente, para el problema de la degradación del suelo, el aumento de las precipitaciones es lo que más ha afectado. Como se explica en el documento: Cambio climático y agricultura en la argentina aspectos institucionales y herramientas de información para la formulación de políticas:

En casi todo el país, hubo un aumento de las precipitaciones medias anuales con mayor incidencia en el noreste y en el centro del país. Este cambio implicó que, por un lado, se facilite la expansión de la frontera agrícola en la zona oeste periférica a la región húmeda tradicional, pero, por otro lado, condujo al anegamiento permanente o transitorio de gran cantidad de campos productivos... El aumento de la frecuencia de inundaciones generó graves consecuencias socioeconómicas en los valles de los grandes ríos de las provincias ubicadas en el este del país. Cabe destacar que, a su vez, se registró un considerable aumento de la frecuencia de precipitaciones extremas en gran parte del este y centro del país con los consiguientes daños por las inundaciones, vientos destructivos y granizo asociados a estos eventos. (S. de Obschatko, 2015, p.32).

Una de las problemáticas que varios productores comentaron, fue el caso de las heladas, explicando que antes en el invierno la cantidad de heladas que se presentaban por temporada estaba entre 25 y 45 veces, mientras que ahora año 2017, se habían presentado tan solo tres. Como consecuencia de esto, fue aumentando el número de moscas afectando el cultivo; en épocas anteriores las heladas podían llegar a controlar las malezas, además de ayudar a permeabilizar el suelo. Así es descrito por uno de los entrevistados:

"No solo las heladas controlaban las malezas, sino que, a su vez, lograban permeabilizar el suelo. Porque la helada se produce por la humedad de la tierra, es decir, toma la humedad de la tierra, se congela y después se evapora, produciendo una permeabilidad del suelo natural, entonces vos llegabas con un control de maleza, con un suelo permeable, que a su vez se mineralizaba por el hecho de tener mayor vínculo con el aire... teníamos casi una fertilidad propia que ahora ya no la tenemos". (Productor A, Cuenca Arroyo Estacas, Entre Ríos, Argentina, 20 de octubre de 2017).

De tal forma, que la reducción en el número de heladas por año estaría provocando el aumento en las dosis de agroquímicos, tanto para fertilizar como para acabar con las plagas. En definitiva, el aumento del clima ha posibilitado el incremento de malezas, que 
antes se controlaba con las heladas, y la presencia de temperaturas más tropicales antes de tiempo, generando comportamientos que a su vez afectan el ambiente. "Una de las características del cambio climático es que se exacerban los extremos. Así como tenés muchos períodos de poca lluvia, tenés otros días con lluvia intensa. Aumentaron los extremos de temperatura y de precipitación", afirma Matilde Rusticucci, investigadora del CONICET y profesora de la Universidad de Buenos Aires UBA en Ciencias de la Atmósfera (Pérez, 2018).

Por lo cual, cuando se habla de la relación entre cambio climático, agricultura y degradación del suelo, lo que se puede apreciar es que en efecto, si se refiere a que el cambio climático es un proceso a nivel global, que se expresa en el aumento de la temperatura en toda la atmósfera de la tierra, su relación con el problema de la degradación del suelo es evidente gracias a que este tiene que ver directamente con la dinámica del agua y en cómo el suelo puede recibir o almacenar más o menos cantidad de agua, entonces un aumento de las precipitaciones, más una baja cobertura arbustiva, agravan la dinámica erosiva de dichos suelos. Por otro lado, si aumenta la temperatura y disminuyen la cantidad de heladas el suelo pierde permeabilidad, en ese caso al llover, igualmente, el suelo no tiene la capacidad de contener agua, agudizando de igual forma este problema.

Entre las entrevistas que se hicieron durante el trabajo de campo, frente a esta situación se encontraron diferentes posiciones: está la posición que asegura que actualmente estamos experimentando un cambio climático, el cual es causado mayormente por actividades humanas y que zonas como la cuenca arroyo Estacas, sufren las consecuencias de este fenómeno. Mientras que hay otras posiciones que manifiestan que, aunque evidentemente el clima se ha modificado sustancialmente en el último siglo, esta situación puede ser originada por ciclos naturales a los que la tierra ha estado sometida a lo largo de su historia. Este punto de vista es relatado por uno de los técnicos entrevistados, en donde expone:

"El año pasado hubo una manga de piedra que se extendió por ciento cincuenta kilómetros y quince de ancho, que no dejó nada, hasta la cáscara de los árboles se cayeron, casa que pasaba le rompía el techo, no había vidrios que resistieran y eso no lo habíamos visto. Y lo mismo, las intensidades de lluvias, es cierto, son más intensas y eso por supuesto le hace más daño al suelo, es decir, que eso es cierto. Vuelvo a lo mismo, lo que yo no sé y seguramente nadie sabe, es si son ciclos de cien años, doscientos o mil o sí cambió realmente el clima". (Técnico A, Cuenca Arroyo Estacas, Entre Ríos, Argentina, 19 de octubre de 2017).

En este caso, si los registros paleo climáticos son certeros, se estaría aseverando que en efecto está ocurriendo un fenómeno el cual llamamos cambio climático, por lo tanto, al ser un fenómeno global afectaría a todo el planeta, incluyendo regiones como la cuenca arroyo Estacas. Esto es importante anotarlo, porque algunos testimonios cuando cuestionaban la relación del cambio climático con el problema de degradación del suelo manifestaban que los efectos del cambio climático podían afectar a unos lugares y a otros no, y que para el caso del problema específico del arroyo de la cuenca Estacas, el problema de degradación ocurría exclusivamente por la actividad agrícola y agropecuaria, y que no se relacionaba con el cambio climático.

Mientras que, por el contrario, argumentos como el de Ana Beatriz Wingeyer técnica del INTA confirman la relación entre cambio climático, la agricultura y el problema de degradación, al explicar cómo el cambio climático afecta la protección del suelo: 
48|El cambio climático y la degradación del suelo

"Lo que está ocurriendo, es que estamos teniendo cambios en los regímenes de temperaturas que hacen que los cultivos estén sufriendo más estrés, más estrés térmico, más estrés hídrico también debido a la temperatura, pero eso hace que produzcan menos biomasa, que rindan menos, por ello hay menos desarrollo de las raíces, lo que sería la agregación de suelo y el desarrollo a una estructura, pero también hay menos producción de la parte aérea de la planta, por lo cual hay menos cobertura del suelo, entonces el cambio climático también afecta a la protección del suelo a través de una menor masa”. (Entrevista a Ana Beatriz Wingeyer, Cuenca Arroyo Estacas, Entre Ríos, Argentina, 10 de octubre de 2017).

Evidentemente, las actividades agrícolas y agropecuarias han sido uno de los causantes por los cuales se han incrementado los problemas de degradación del suelo, pero además de esto, tales actividades humanas han aportado en el aumento de emisiones de dióxido de carbono, óxido nitroso, metano, distintos gases de efecto invernadero, entre otros, modificando radicalmente el clima y que al ser modificado promueve cambios que terminan por afectar a la tierra. Es decir, directa o indirectamente, al final el cambio climático termina relacionándose con esta problemática. Como se describe en el artículo Género, infancia y antropología frente al cambio climático "El aspecto local, social y cultural del cambio climático subraya y resalta todas las contradicciones inherentes a las sociedades y hace inciertas y contradictorias muchas soluciones posibles (o propuestas)" (Breda, 2019).

\section{Conclusión}

Analizando la situación del problema de la degradación del suelo en la cuenca arroyo Estacas, se puede concluir que son varios los factores que han influido en esta situación. En primer lugar, existe una predisposición natural del suelo a erosionarse, debido al tipo de suelos y a la forma del paisaje, en segundo lugar, durante mucho tiempo ha existido un inadecuado uso del recurso suelo por parte del hombre. Entre las principales actividades que se han desarrollado en la cuenca y que han influido en la degradación, se encuentra: el mal manejo ganadero, la expansión de la frontera agrícola y la deforestación. En el caso del mal manejo ganadero, este perjudica el suelo especialmente porque algunos campos sobrepasan la capacidad de carga, es decir, hay más cantidad de cabezas de ganado por hectárea de las que deberían estar, en donde el pisoteo constante de los animales va reduciendo la capa herbácea aumentando la erosión.

La expansión agrícola, representada especialmente en el aumento del cultivo de soja, aunque también en la producción de cultivos como lino, maíz y trigo, entre otros, los cuales a través del tiempo desarrollaron prácticas productivas que influyeron significativamente en el desgaste del suelo. Igualmente, para el caso de los productores agrícolas que tienen en alquiler o arrendamiento los campos, la presión sobre el recurso del suelo es más acentuado, produciendo que los procesos de degradación sean más intensos. Por último, la deforestación, que, relacionada con el aumento de la frontera agrícola, también ha incidido en esta situación.

En cuanto al cambio climático, si realmente se relaciona con la degradación del suelo, también estaría influyendo en este problema. Por lo tanto, aunque la mayoría de los especialistas consultados, coinciden en que un fenómeno extremo aislado como la sequía o las inundaciones no son consecuencia directa del cambio climático, el aumento en la frecuencia y en la intensidad de estos sí lo son. El comportamiento del clima, se ha vuelto impredecible y su variabilidad se agiganta, al igual que el impacto sobre el sector agropecuario (Pérez, 2018). Por el momento, existe un acuerdo entre los especialistas en el tema, 
ya que debido a la intervención del ser humano los cambios en el clima evidentemente se han acelerado, modificando así los ciclos naturales experimentados en el último siglo.

\section{Referencias Bibliográficas}

Aranda, A. (2014, 13 de diciembre). Evaluando la desertificación en las tierras riojanas. CONICET. Recuperado de https://www.conicet.gov.ar/evaluando-la-desertificacion-enlas-tierras-riojanas/ fecha de consulta: 12/06/2019.

Ayestarán, I. (2004). De la historia y la sociología ambientales a la ecología política: factores tecnocientíficos, sociohistóricos y ecosistémicos en la investigación sobre el cambio climático. Norba: Revista de Historia, (17), 111-134. Recuperado de https://dialnet.unirioja.es/servlet/articulo?codigo $=1158937$.

Breda, N. (2019). Género, infancia y antropología frente al cambio climático. Collectivus, Revista De Ciencias Sociales, 6(1), 197-214. DOI: https://doi.org/10.15648/ Coll.1.2019.11

Casas, R., \& Albarracín, G. (2015). El deterioro del suelo y del ambiente en la Argentina (Tomo 2). Buenos Aires: INTA-PROSA.

Caviglia, O., Ana B. W., \& Leonardo N. (2016). El rol de los suelos agrícolas frente al cambio climático. Serie de Extensión INTA Paraná, (78), 27-32.

Dupleich, J. (2012). Una experiencia de desarrollo territorial con historia en las colonias del norte del departamento La Paz. INTA. Revista Experiencias de extensión actuando en los territorios, (67), 36-90.

Dupleich, J., \& Vicente, G. (2012). Importancia de la agricultura familiar en el departamento La Paz. INTA. Revista Experiencias de extensión actuando en los territorios, (67), 150-170.

Encina, A., \& Ibarra, J. (2003). La degradación del suelo y sus efectos sobre la población. Revista Población y Desarrollo, (5), 5-10.

Esteban, M., Amador, L., Mateos, F., y Olmedo, F. (2019). Mujer y medio ambiente. Una aproximación desde la acción socioeducativa. Collectivus, Revista De Ciencias Sociales, 6(1), 177-195. DOI: https://doi.org/10.15648/Coll.1.2019.10

Leff, E. (2010). Sustentabilidad y racionalidad ambiental: hacia "otro" programa de sociología ambiental. Revista Mexicana de Sociología, 73(1), enero-marzo. Recuperado de http://www.scielo.org.mx/scielo.php?script=sci_arttext\&pid=S018825032011000100001

Lezana, L., Litwin, G., Mancuso, W. \& Pueyo, J. (2012). Los sistemas ganaderos en el área de influencia de INTA EEA Paraná. INTA. Revista Experiencias de extensión actuando en los territorios, (67), 89-112.

Natenzon, C., y Tito, G. (2001). Medio ambiente y pequeños productores. Argentina: Secretaría de Agricultura, Ganadería, Pesca y Alimentación. Recuperado de http://www.fao.org/family-farming/detail/en/c/318251/ 
$\mathbf{5 0} \mid$ El cambio climático y la degradación del suelo

Pérez, V. (2018, 13 de agosto). Este es el nuevo clima. Recuperado de https://www.portalechero.com/innovaportal/v/13435/1/innova.front/este-es-el-nuevoclima.html

S. de Obschatko, E. (2015). Cambio climático y agricultura en la Argentina: aspectos institucionales y redes de información para la formulación de políticas. Buenos Aires: Instituto Interamericano de Cooperación para la Agricultura IICA.

Sommantico, S. (2018, 19 de febrero). La huella que deja el cambio climático en la agricultura. Recuperado de https://www.infocampo.com.ar/la-huella-que-deja-elcambio-climatico-en-la-agricultura/ 